\title{
Enhanced High Oxygen Permeation of Mixed-Conducting Multichannel Hollow Fiber Membrane via Surface Modified Porous Layer
}

\author{
Shaobin Guo, Jiawei Zhu, Zhengkun Liu, Xin Jiang, Zhicheng Zhang, and Wanqin Jin* \\ State Key Laboratory of Materials-Oriented Chemical Engineering, College of Chemistry and Chemical Engineering, Nanjing Tech \\ University, 5 Xinmofan Road, Nanjing 210009, P. R. China
}

ABSTRACT: The oxygen permeation performance of $\mathrm{Ba}_{0.5} \mathrm{Sr}_{0.5} \mathrm{Co}_{0.8} \mathrm{Fe}_{0.2} \mathrm{O}_{3-\delta}$ (BSCF) mixed-conducting multichannel hollow fiber (MCMHF) membranes was improved by surface modification via spin-spraying of a $\mathrm{La}_{0.6} \mathrm{Sr}_{0.4} \mathrm{CoO}_{3-\delta}$ (LSC) porous layer. At $1173 \mathrm{~K}$, the oxygen permeation flux of the modified membranes was clearly enhanced and reached $9.68 \mathrm{~mL} \cdot \mathrm{cm}^{-2} \cdot \mathrm{min}{ }^{-1}$, which is a remarkable high value in the field of mixed-conducting oxygen permeation membrane processes. Theoretical calculations demonstrated that the oxygen transport resistance, especially the surface exchange resistance, obviously decreased as a result of the modified LSC porous layer. Moreover, the process of oxygen permeation through the modified membrane was controlled by both bulk diffusion and the surface oxygen exchange reaction, whereas the oxygen permeation of the unmodified membrane was dominantly controlled by the surface oxygen exchange reaction. The modified MCMHF membranes showed generally stable oxygen permeation fluxes over $100 \mathrm{~h}$ at $1173 \mathrm{~K}$.

\section{INTRODUCTION}

Mixed-conducting membrane processes have attracted considerable interest because of their wide potential applications in oxygen separation from air, ${ }^{1-3} \mathrm{CO}_{2}$ capture and storage, ${ }^{4,5}$ catalytic membrane reactors, ${ }^{6-8}$ and solid oxide fuel cells. ${ }^{9,10}$ Among the applications, separating oxygen from air is one of the most promising membrane processes. ${ }^{11-13}$ In addition, compared with conventional cryogenic air separation and the pressure swing adsorption process, mixed-conducting membranes possess many distinct advantages such as easy operation, energy savings, and economic benefits. For example, oxygenenriched air or pure oxygen produced by a mixed-conducting membrane can be used in the combustion process instead of air, which will lead to a significant increase in fuel efficiency, resulting in a decrease of $\mathrm{CO}_{2}$ emissions. ${ }^{12,14}$ However, despite the advantages of using mixed-conducting membranes to separate oxygen from air, there are some challenges of configuring this technology in large-scale applications, such as improving the oxygen permeability, long-term stability, and mechanical strength. To solve these issues, much research has been directed toward optimization of the membrane configuration and the development of novel mixed-conducting membrane materials with high performance.

From a theoretical point of view, oxygen transfer through a mixed-conducting membrane consists of three progressive steps: (1) decomposition of oxygen molecules on the feed side; (2) simultaneous bulk diffusion of electron holes and oxygen vacancies or ions; and (3) recombination to form oxygen molecule on the permeate side. The slowest process or the slowest-moving species is deemed to be the rate-limiting factor for the oxygen permeation process. In general, when the wall of a dense membrane is thick enough, the resistance across the membrane is proportional to its thickness. Therefore, oxygen permeation is limited by the bulk diffusion. ${ }^{15}$ On these premises, decreasing the thickness of the dense membrane layer can be considered as an effective method to improve the oxygen permeation flux. Over the past few decades, three main membrane configurations, disc-shaped, tubular, and hollow fiber, have been employed to optimize the effective route for the oxygen permeation process. Disc-shaped membranes can be easily fabricated via the conventional static pressing method. However, many obstructions have been encountered when taking into account the industrialization, such as sealing and module connection. In addition, dense tubular membranes fabricated by cold isotactic pressing or plastic extrusion have thick membrane walls and low surface/volume ratios. Currently, hollow fiber membranes that possess an asymmetrical microstructure are being increasingly noticed and applied. Besides, compared with the disc and tubular techniques, the hollow fiber membranes provide thin separating layers with high oxygen permeation fluxes and possess the largest membrane area per unit packing volume. Nevertheless, suffering from low mechanical strength, the conventional hollow fiber membranes may not meet the industrial demands.

When the membrane is sufficiently thin, the oxygen transfer resistance mainly lies in the oxygen molecular reaction at the membrane surface. Because of this, some efforts can be made to improve the surface oxygen exchange reaction to enhance the oxygen permeation flux. This can be achieved by (i) roughening the membrane surface by certain means ${ }^{16}$ or coating the membrane with a porous layer, ${ }^{17}$ which increases the effective surface area of the membranes, or (ii) modifying the membrane surface with materials possessing properties that accelerate the oxygen exchange reaction. ${ }^{11,18,19}$ In our very recent work, mixed-conducting multichannel hollow fiber (MCMHF)

Received: March 16, 2015

Revised: June 16, 2015

Accepted: June 22, 2015 
membranes with a sufficiently thin wall, as well as high oxygen permeation flux and high mechanical strength, were proposed. ${ }^{20}$ Subsequent comprehensive experiments demonstrated that the surface reaction was the rate-limiting step for oxygen transport. ${ }^{21}$ Therefore, enhancement of the oxygen permeation fluxes of the MCMHF membranes could be achieved by surface modification.

In this work, MCMHF membranes based on the state-of-theart material $\mathrm{Ba}_{0.5} \mathrm{Sr}_{0.5} \mathrm{Co}_{0.8} \mathrm{Fe}_{0.2} \mathrm{O}_{3-\delta}$ (BSCF) with an ultrathin dense layer were prepared by combining a phase inversion and sintering techniques. ${ }^{9} \mathrm{La}_{0.6} \mathrm{Sr}_{0.4} \mathrm{CoO}_{3-\delta}$ (LSC) was chosen as the catalytic material to modify the membrane surfaces because it can produce high concentrations of oxygen ion vacancies and then facilitate oxygen ion transport. ${ }^{22,23}$ The porous LSCmodified layer was achieved by a spin-spraying process. In this work, the increase in the oxygen permeation flux, the transformation of the rate-limiting steps before and after surface modification, the effects of various thicknesses of the modified layer on the oxygen flux, and the long-term stability of the modified membrane were investigated systematically.

\section{EXPERIMENTAL SECTION}

2.1. Fabrication of the Samples. The $\mathrm{Ba}_{0.5} \mathrm{Sr}_{0.5} \mathrm{Co}_{0.8} \mathrm{Fe}_{0.2} \mathrm{O}_{3-\delta}$ powder was synthesized via solid-state reaction techniques. The required stoichiometric amounts of $\mathrm{BaCO}_{3}$ (99\%), $\mathrm{SrCO}_{3}$ (99\%), $\mathrm{Co}_{2} \mathrm{O}_{3}$ (99\%), and $\mathrm{Fe}_{2} \mathrm{O}_{3}$ (99\%) were mixed, then ball-milled in ethanol for $24 \mathrm{~h}$, and then dried at $393 \mathrm{~K}$. The BSCF oxide was calcined at $1223 \mathrm{~K}$ for $5 \mathrm{~h}$ with heating and cooling rates of $2 \mathrm{~K} / \mathrm{min}$ in air. The sintered BSCF powder was ground and sieved (300 mesh) to prepare the MCMHF membrane. Similarly, the required stoichiometric amounts of $\mathrm{La}_{2} \mathrm{O}_{3}$ (99\%), $\mathrm{SrCO}_{3}(99 \%)$, and $\mathrm{Co}_{2} \mathrm{O}_{3}$ (99\%) were used to synthesize $\mathrm{La}_{0.6} \mathrm{Sr}_{0.4} \mathrm{CoO}_{3-\delta}$ powder as described above.

The combination of phase inversion and a sintering process was used to prepare the green multichannel hollow fiber. The spinning solution was composed of 61.68 wt \% BSCF powder, 30.80 wt \% 1-methyl-2-pyrrolidinone (NMP), 6.91 wt \% polyethersulfone (PESf), and $0.61 \mathrm{wt} \%$ polyvinylpyrrolidone (PVP). As shown in Figure 1 the multichannel hollow fiber

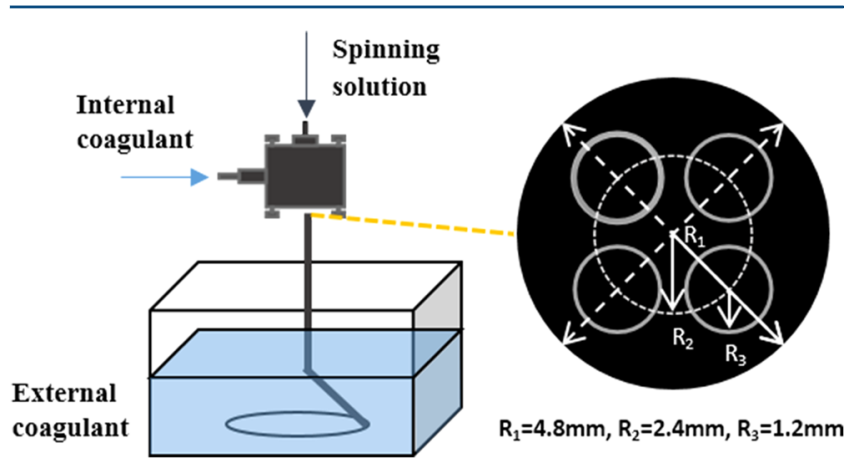

Figure 1. Schematic diagram of the phase inversion. The inset shows a picture of the multichannel hollow fiber spinneret used in this work.

precursors obtained using a tetrabore spinneret with an orifice diameter of $4.8 \mathrm{~mm}$ and four bores with diameters of $1.2 \mathrm{~mm}$. Tap water and deionized water were applied as the external and internal coagulants, respectively. Afterward, the membrane precursors were calcined at $1373 \mathrm{~K}$ for $5 \mathrm{~h}$.

The LSC layer was formed on the outside surface of a polished MCMHF membrane by a spin-spraying method. ${ }^{24} \mathrm{~A}$ slurry of 8.41 wt \% LSC powder (sieved through a 300 mesh sieve), 66.25 wt \% isopropyl alcohol, 18.91 wt \% ethylene glycol, and 6.43 wt \% glycerin was used as the spraying precursor. The dense and crack-free MCMHF membrane was rotated around the central axis at $200 \mathrm{rpm}$, and simultaneously, a spraying gun moved along the horizontal axis at $30 \mathrm{~cm} \cdot \mathrm{min}^{-1}$. At $393 \mathrm{~K}$, the slurry was sprayed on the rotating MCMHF membrane from the spraying gun at a rate of $2 \mathrm{~mL} \cdot \mathrm{min}^{-1}$, and upon evaporation of the alcohols, the LSC powder was deposited on the membrane surface. The green LSC-modified BSCF membrane was sintered at $1273 \mathrm{~K}$ for $2 \mathrm{~h}$ to burn out the organic solvents and generate the porous LSC layer.

2.2. Characterization. X-ray diffraction (XRD) with $\mathrm{Cu}$ $\mathrm{K} \alpha$ radiation (Bruker, model D8 Advance, Germany) was used to analyze the phase structures of the BSCF, LSC powder, and the BSCF hollow fiber membrane. The diffraction patterns were collected by step scanning over the $2 \theta$ range from $20^{\circ}$ to $80^{\circ}$ with an increment of $0.05^{\circ}$ at room temperature. The morphology and microstructure of the unmodified and modified membranes were analyzed by scanning electron microscopy (SEM) using an S-4800 scanning electron microscope (Hitachi, Tokyo, Japan). The gas tightness of the BSCF and LSC-modified BSCF membranes was examined using nitrogen at room temperature. In detail, we first placed the membranes into a homemade module that was similar to the oxygen permeation device, and then nitrogen at 2 atm absolute pressure was introduced into the lumen side. If no permeation of nitrogen was detected on the permeation side of the membrane, the membrane could be regarded as gas-tight.

2.3. Oxygen Permeation Measurements. The measurement of oxygen permeation fluxes through the unmodified and modified membranes was performed using a high-temperature oxygen permeation apparatus. ${ }^{25} \mathrm{~A}$ dense and crack-free membrane with a length of $20 \mathrm{~mm}$ was sealed between two alumina tubes (i.d. $=2.5 \mathrm{~mm}$, o.d. $=4 \mathrm{~mm}$ ) using silver adhesive. The two alumina tubes surrounded by a quartz tube (i.d. $=8 \mathrm{~mm}$, o.d. $=12 \mathrm{~mm}$ ) formed the shell side. Air was introduced into the shell side while helium was swept on the permeation side to take the permeated oxygen away from the apparatus. The gaseous mixture from the permeation side was analyzed using an online gas chromatograph (model GC-8A, Shimadzu, Kyoto, Japan). Both the feed-side and sweep-gas flow rates were regulated by mass flow controllers. The leakage of oxygen through the sealant was less than $0.5 \%$ of the total oxygen flux during the experiment, indicating that its effect on the oxygen permeation can be ignored.

\section{RESULTS AND DISCUSSION}

The XRD patterns of the LSC powder, BSCF membrane, and BSCF powder sintered under different temperatures are shown in Figure 2. They indicate that both the BSCF powder and membrane showed strong diffraction peaks assigned to the cubic lattice structure. However, the BSCF membrane contained a trace amount of $\mathrm{BaSO}_{4}$ phase, which could be observed in the XRD patterns at $2 \theta \approx 26.2,27.3,29.2$, and $42.9^{\circ}$. These impurity peaks arose from the use of sulfurcontaining binders. ${ }^{26}$ The sample of LSC powder also showed a perovskite phase structure with all of the diffraction peaks assigned to the orthorhombic phase. In general, LSC powder, BSCF powder, and the sintered membrane all maintained perovskite crystalline structures.

The overall micrographs of the BSCF MCMHF membranes before and after modification with a porous LSC layer are 


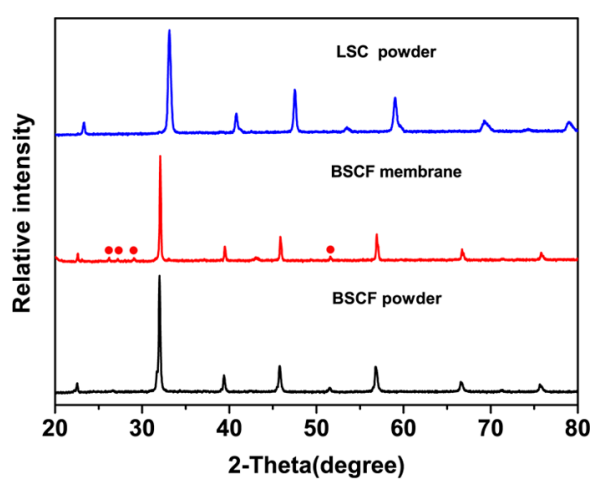

Figure 2. XRD patterns of the synthesized BSCF powder, BSCF membrane, and LSC powder.

shown in Figure 3. The sintered membrane had a well-formed tetrabore structure with outer and channel diameters of approximately 2.3 and $0.8 \mathrm{~mm}$, respectively (Figure 3a). The fabricated MCMHF membrane has a typical asymmetric structure with fingerlike porous layers near the exterior and
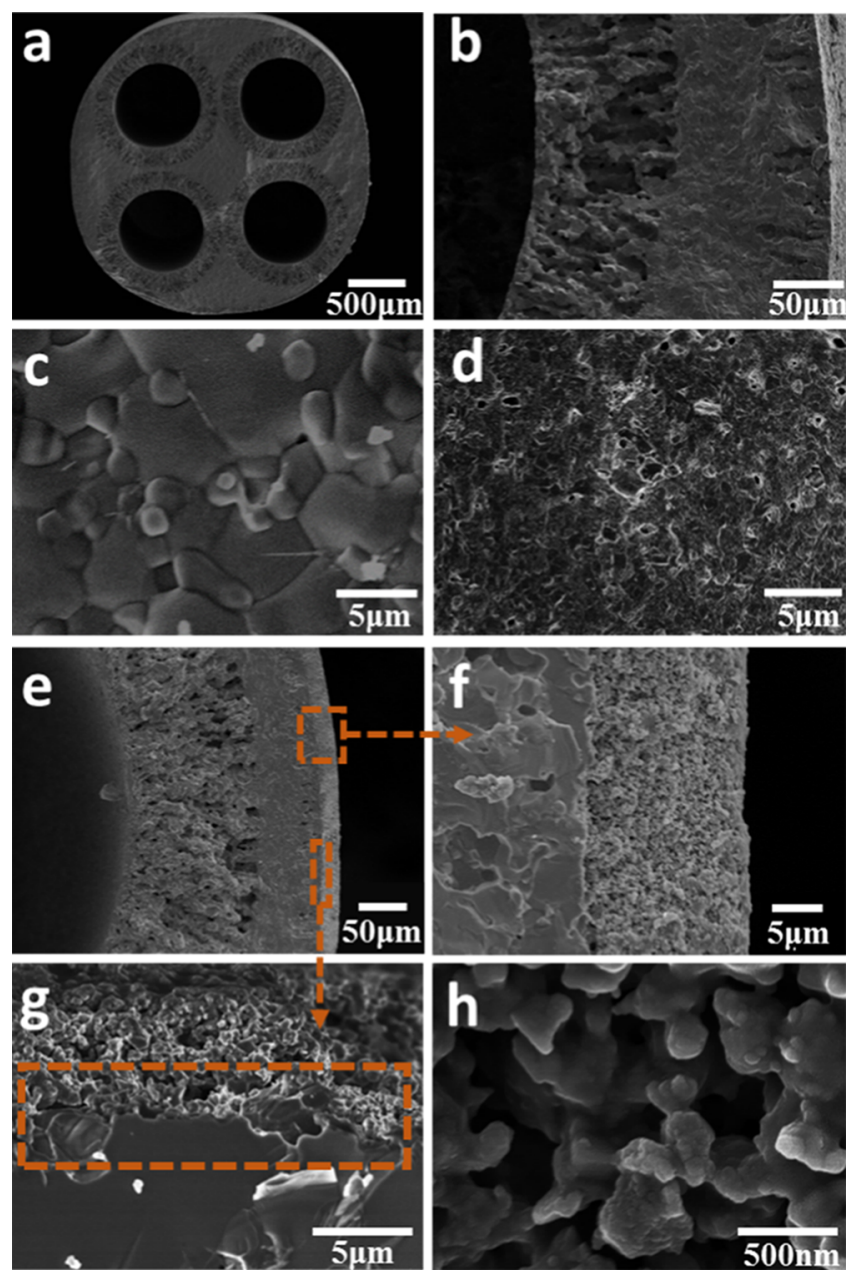

Figure 3. SEM images of the uncoated and LSC-coated BSCF hollow fiber membranes: (a) cross section of the uncoated BSCF hollow fiber membrane; (b) wall of the uncoated membrane; (c) outer surface of the uncoated membrane; (d) inner channel surface of the uncoated membrane; (e) wall of the LSC-coated membrane; (f) cross-section of the LSC-coated layer; (g) interface of the BSCF and LSC layers; (h) surface of the LSC-coated layer. channel surfaces (Figure 3b). The formation of the asymmetric structures is caused by the intricate interactions among the solvent, nonsolvent, and binder during the phase inversion process. The dense, thin layer can result from the rapid precipitation that occurred on the outer layer of the precursor, while the slow precipitation gave the porous, spongelike structure. After sintering, the spongelike structure of the precursor formed a dense layer at the center of the wall of hollow fiber. Figure $3 b$ shows that the thickness of the thinnest part of the dense layer is about $50 \mu \mathrm{m}$ between the exterior and channel surface. The micrograph in Figure $3 \mathrm{c}$ shows that the exterior surface is completely densified, whereas some irregular pinholes are observed on the channel surface (Figure $3 \mathrm{~d}$ ). These pinholes are the opening ends of the fingerlike pores near the channel surface, which would enhance the surface oxygen exchange reactions on the sweep side by promotion of the transfer of the lattice oxygen to molecular oxygen. From the cross-sectional image of the membrane (Figure $3 \mathrm{e}$ ), it can be seen that the bulk dense layer and the LSC porous layer were closely combined with each other. The thickness of the modified layer was approximately $18 \mu \mathrm{m}$, as obtained from Figure 3f. Because a well-formed integration between two different phases is of great importance to decrease the boundary oxygen ion transport resistance and surface oxygen exchange, ${ }^{27}$ the good integration layer at the boundary of the two phases (Figure 3g), LSC and BSCF, will be useful to improve the oxygen permeation performance. Figure $3 \mathrm{~h}$ displays the surface morphology of the LSC porous layer, showing that the membrane surface was composed of granular ceramic particles.

In Figure 4a, the oxygen permeation fluxes of the BSCF membranes with and without surface modification are plotted as functions of temperature. The curves show that the modified membrane exhibited an ultrahigh oxygen permeation flux, which is nearly 3 times that of the unmodified membrane. For example, at $1173 \mathrm{~K}$, the oxygen permeation flux of the unmodified membrane was $3.71 \mathrm{~mL} \cdot \mathrm{cm}^{-2} \cdot \mathrm{min}^{-1}$, whereas it was $9.68 \mathrm{~mL} \cdot \mathrm{cm}^{-2} \cdot \mathrm{min}^{-1}$ after surface modification. The dramatic enhancement confirmed that the modified LSC layer plays a significant role in increasing the oxygen flux of BSCF hollow fiber membranes. Two reasonable explanations have been proposed for this enhancement. First, the modified membrane with a porous layer can enlarge the effective contact area of the surface exchange reaction. Second, the high concentrations of oxygen ion vacancies produced by the LSC layer can promote the surface oxygen exchange rate. Figure $4 \mathrm{~b}$ shows the accurate Arrhenius activation energies of these hollow fiber membranes. The activation energy of the LSCmodified membranes $(35.41 \mathrm{~kJ} / \mathrm{mol})$ was lower than that of the unmodified ones $(46.14 \mathrm{~kJ} / \mathrm{mol})$, indicating that the resistance of the oxygen exchange reaction decreased after the surface modification. Since the overall driving force across the membrane was generally unchanged, this result clearly demonstrated that the porous LSC layer changed the ratelimiting step of the oxygen transport process across the BSCF multichannel hollow fiber membrane.

Figure 5 shows the oxygen permeation fluxes of the unmodified and modified membranes as functions of the air flow rates and sweep helium flow rates at different temperatures. As shown in Figure 5a, the oxygen permeation flux increased with increasing feed-air flow rate over the 1123-1173 $\mathrm{K}$ range, suggesting that the feed air should be abundantly provided to the operation units in order to obtain a higher oxygen permeation flux. ${ }^{21}$ Figure $5 \mathrm{~b}$ illustrates the influence of 
a)

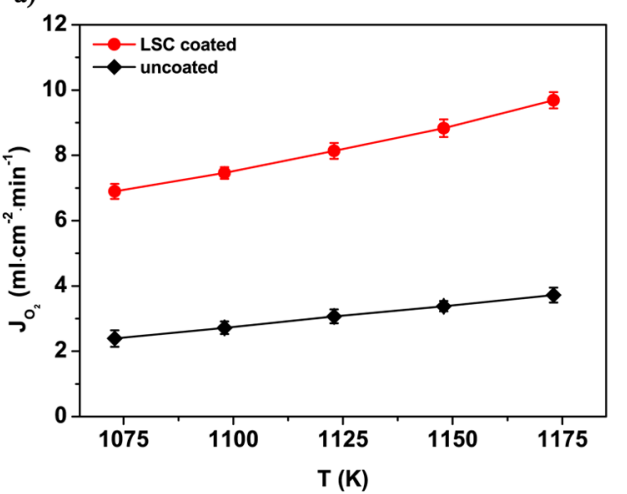

b)

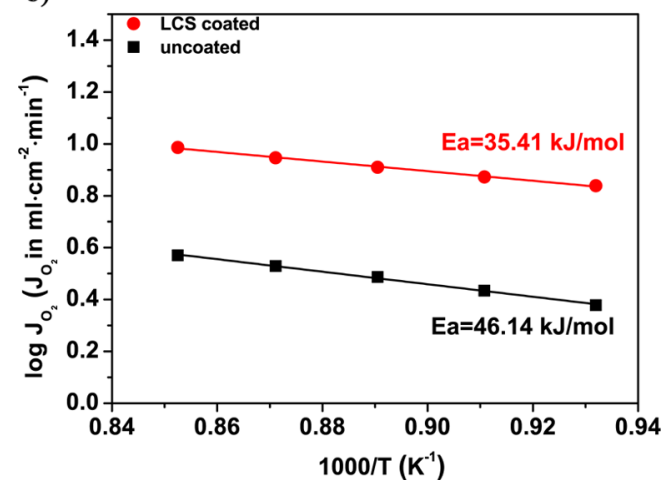

Figure 4. (a) Comparison of oxygen permeation fluxes through the uncoated and LSC-coated membranes at different temperatures. (b) Arrhenius plots of the oxygen permeation fluxes of the uncoated and LSC-coated membranes at different temperatures. $\left(F_{\text {air }}=120 \mathrm{~mL}\right.$. $\left.\min ^{-1}, F_{\mathrm{He}}=80 \mathrm{~mL} \cdot \mathrm{min}^{-1}\right)$.

varying the sweeping gas flow on the oxygen permeation flux at various temperatures. As expected, a higher oxygen permeation flux was obtained when a higher helium flow rate lowered the oxygen partial pressure on the sweep side and increased the driving force for oxygen permeation. For instance, at $1123 \mathrm{~K}$, when the sweep-gas flow rate increased from 20 to $80 \mathrm{~mL}$. $\mathrm{min}^{-1}$, the oxygen permeation fluxes through the unmodified and modified membranes were dramatically improved from 1.58 to $3.21 \mathrm{~mL} \cdot \mathrm{cm}^{-2} \cdot \mathrm{min}^{-1}$ and 4.01 to $8.13 \mathrm{~mL} \cdot \mathrm{cm}^{-2} \cdot \mathrm{min}^{-1}$, respectively. Also, the corresponding oxygen permeation fluxes varied from 1.82 to $3.72 \mathrm{~mL} \cdot \mathrm{cm}^{-2} \cdot \mathrm{min}^{-1}$ and 4.71 to $9.68 \mathrm{~mL}$. $\mathrm{cm}^{-2} \cdot \mathrm{min}^{-1}$, when the temperature increased to $1173 \mathrm{~K}$. To illustrate the reasons for this phenomenon, the influence of the sweep helium flow rate on the oxygen partial pressure on the feed side and the sweep side of the modified membranes at different temperatures was investigated, and the results are shown in Figure 6. The oxygen partial pressure on the sweep side decreased a lot with increasing sweep-gas flow rate, while the value of the oxygen partial pressure on the feed side changed a little. Therefore, the high helium flow rate increased the oxygen partial pressure difference across the two sides of the membrane. According to eq 1, the increase in the oxygen partial pressure difference caused by the increased helium flow rate can enhance the driving force for oxygen transport and thus increase the oxygen permeation flux.

The influence of the oxygen partial pressure of the feed gas on the oxygen permeation fluxes through the unmodified and modified membranes was studied at 1123 and $1173 \mathrm{~K}$. The total flow rate of the feed gas was maintained at $120 \mathrm{~mL} \cdot \mathrm{min}^{-1}$. Different oxygen partial pressures were achieved via the
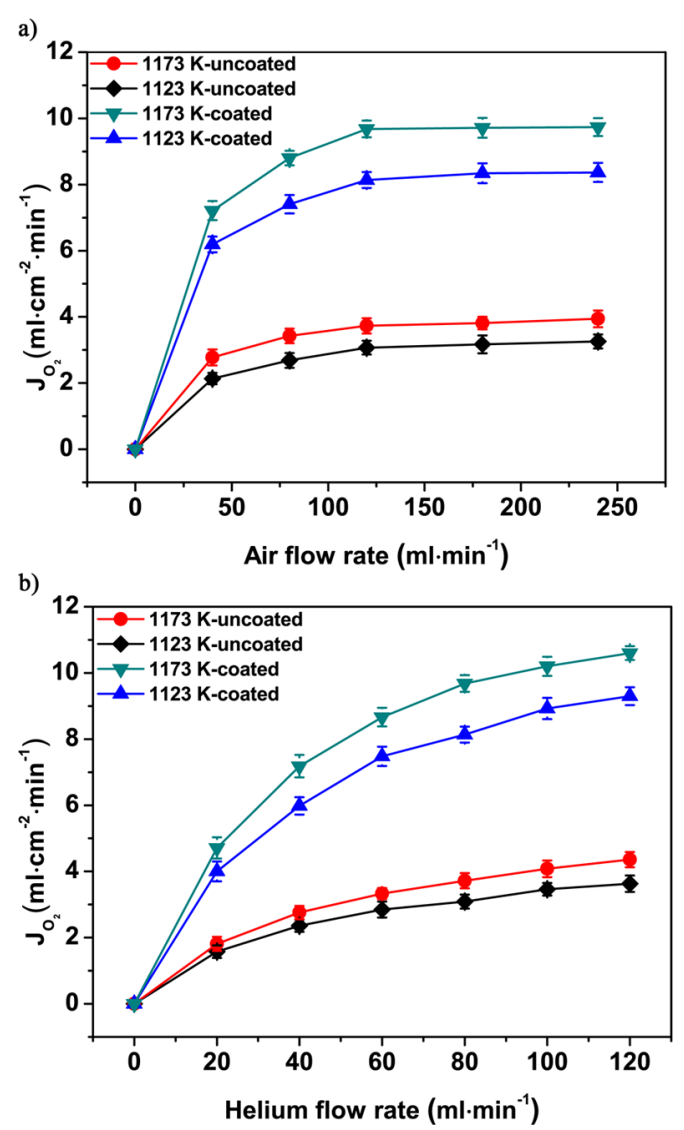

Figure 5. Effects of the air flow rate and helium sweep rate on the oxygen fluxes through the coated and uncoated multichannel hollow fiber membranes at 1123 and $1173 \mathrm{~K}$ : (a) air flow rate $\left(F_{\mathrm{He}}=80 \mathrm{~mL}\right.$. $\left.\mathrm{cm}^{-2} \cdot \mathrm{min}^{-1}\right)$; (b) helium flow rate $\left(F_{\text {air }}=120 \mathrm{~mL} \cdot \mathrm{cm}^{-2} \cdot \mathrm{min}^{-1}\right)$.

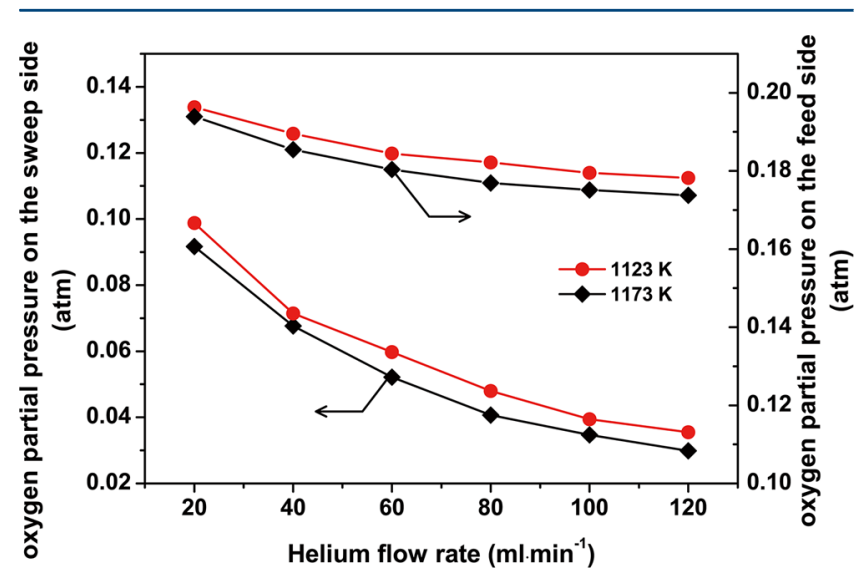

Figure 6. Effects of helium flow rates on the oxygen partial pressures on both sides of the LSC-coated BSCF membrane $\left(F_{\text {air }}=120 \mathrm{~mL}\right.$. $\left.\min ^{-1}\right)$.

proportional adjustment of $\mathrm{N}_{2}$ and $\mathrm{O}_{2}$ on the feed side (the helium flux remained at $80 \mathrm{~mL} \cdot \mathrm{min}^{-1}$ on the sweep side). As shown in Figure 7 , because of the enhanced oxygen concentration gradient difference across the membrane, the oxygen permeation fluxes through both the unmodified and modified membranes increased dramatically with the elevated oxygen partial pressure. Typically at $1173 \mathrm{~K}$, the oxygen permeation flux of the modified membrane at 0.8 atm was 20.1 $\mathrm{mL} \cdot \mathrm{cm}^{-2} \cdot \mathrm{min}^{-1}$, which was much higher than the oxygen permeation flux at $0.21 \mathrm{~atm}$. A similar phenomenon was also 


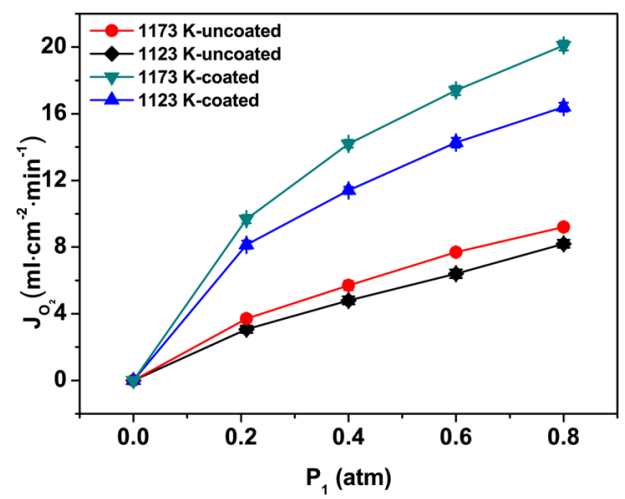

Figure 7. Comparison of oxygen permeation fluxes through the uncoated and LSC-coated hollow fiber membranes as functions of the oxygen partial pressure at $1173 \mathrm{~K}\left(F_{\mathrm{He}}=80 \mathrm{~mL} \cdot \mathrm{min}^{-1}\right)$.

found in the unmodified membrane. Furthermore, the oxygen permeation flux of the modified membrane tends to be more sensitive to the oxygen partial pressure than that of the unmodified membrane because the driving force affects the bulk diffusion process more than the surface exchange kinetics. ${ }^{28}$ In view of the fact that the oxygen permeation flux of the modified MCMHF membrane significantly reached 20.1 $\mathrm{mL} \cdot \mathrm{cm}^{-2} \cdot \mathrm{min}^{-1}$ (under the conditions of an oxygen partial pressure of $0.8 \mathrm{~atm}$ on the feed side and a helium sweep flow rate of $80 \mathrm{~mL} \cdot \mathrm{min}^{-1}$ ), the MCMHF process is a very promising approach in the future industrial production of pure oxygen.

In order to interpret the variations of the oxygen permeation resistance, the dependence of the oxygen permeation flux on the oxygen driving force was studied. As we know, when the oxygen permeation process is controlled by bulk diffusion, the relationship of the oxygen permeation flux $J_{\mathrm{O}_{2}}$ to other parameters can be described by Wagner's equation as follows: ${ }^{29}$

$$
J_{\mathrm{O}_{2}}=-\frac{R T}{4^{2} F^{2} L} \int_{\ln P_{1}}^{\ln P_{2}} \frac{\sigma_{\mathrm{e}} \sigma_{\mathrm{i}}}{\sigma_{\mathrm{e}}+\sigma_{\mathrm{i}}} \mathrm{d} \ln P
$$

where $R$ and $F$ represent the gas constant and the Faraday constant, respectively; $T$ represents the operating temperature; $L$ represents the membrane thickness; $P_{1}$ and $P_{2}$ represent the oxygen partial pressures on the feed and sweep sides, respectively; and $\sigma_{\mathrm{e}}$ and $\sigma_{\mathrm{i}}$ represent the electronic and ionic conductivities, respectively. However, while the oxygen permeation process is controlled by both bulk diffusion and the surface oxygen exchange reaction, the oxygen permeation flux can be expressed by the modified Wagner equation as follows:

$$
J_{\mathrm{O}_{2}}=-\frac{1}{1+\left(2 L_{\mathrm{C}} / L\right)} \frac{R T}{4^{2} F^{2} L} \int_{\ln P_{1}}^{\ln P_{2}} \frac{\sigma_{\mathrm{e}} \sigma_{\mathrm{i}}}{\sigma_{\mathrm{e}}+\sigma_{\mathrm{i}}} \mathrm{d} \ln P
$$

where $L_{\mathrm{C}}$ is the characteristic membrane thickness. ${ }^{30}$ For the BSCF mixed-conducting oxide, $\sigma_{\mathrm{e}} \gg \sigma_{\mathrm{i}}$, so eq 2 can be simplified as follows:

$$
J_{\mathrm{O}_{2}}=-\frac{1}{1+\left(2 L_{\mathrm{C}} / L\right)} \frac{R T}{4^{2} F^{2} L} \int_{\ln P_{1}}^{\ln P_{2}} \sigma_{\mathrm{i}} \mathrm{d} \ln P
$$

Because the ionic conductivity of oxygen is related to the oxygen vacancies, a more general expression can be obtained using the Nernst-Einstein relation: ${ }^{31}$

$$
\sigma_{\mathrm{i}}=\frac{4 F^{2} V_{\mathrm{O}}^{\infty} D_{\mathrm{v}}}{R T V_{\mathrm{m}}}
$$

where $V_{\mathrm{O}}^{\infty}$ represents the concentration of oxygen vacancies, $D_{\mathrm{v}}$ represents the vacancy diffusion coefficient, and $V_{\mathrm{m}}$ represents the perovskite molar volume. Because the vacancy diffusion coefficient is further related to the partial pressure of oxygen $(P), \sigma_{i}$ can follow the relation ${ }^{32}$

$$
\sigma_{\mathrm{i}}=\sigma_{\mathrm{i}}^{0}\left(P / P_{0}\right)^{n}
$$

where $\sigma_{\mathrm{i}}^{0}$ is the oxygen ionic conductivity at $1 \mathrm{~atm}$ and $P_{0}=1$ atm. Combining eq 3 and eq 5 under the condition $\sigma_{\mathrm{e}} \gg \sigma_{\mathrm{i}}$, we obtain the following equation:

$$
\begin{aligned}
J_{\mathrm{O}_{2}} & =\frac{1}{1+\left(2 L_{\mathrm{C}} / L\right)} \frac{R T \sigma_{\mathrm{i}}^{0}}{4^{2} F^{2} L n}\left[\left(P_{2} / P_{0}\right)^{n}-\left(P_{1} / P_{0}\right)^{n}\right] \\
& =\alpha\left[\left(P_{1} / P_{0}\right)^{n}-\left(P_{2} / P_{0}\right)^{n}\right]
\end{aligned}
$$

The rate-limiting step of oxygen permeation through the membrane can be deduced from the value of $n$. Generally, for $n$ $\geq 0.5$, the surface exchange kinetics is rate-limiting; for $n<0$, the bulk diffusion process of the oxygen ion is the main ratelimiting step; and for $0<n<0.5$, the oxygen permeation is controlled by both the surface oxygen exchange reaction and bulk diffusion. ${ }^{27,33,34}$ The optimal linear fits to determine the $n$ values are shown in Figure 8 . The $n$ values obtained from the

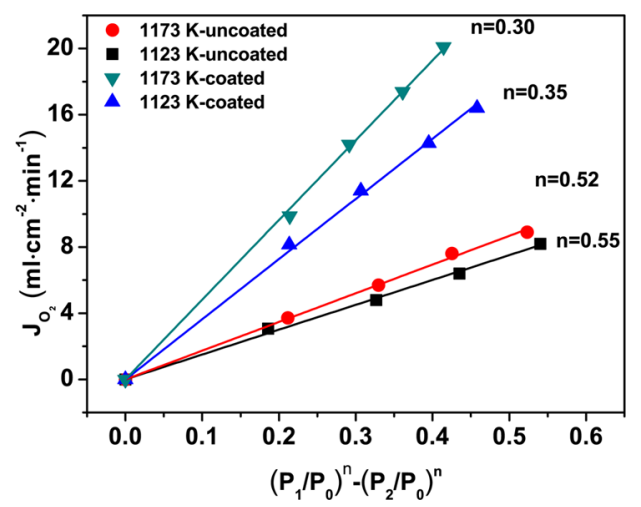

Figure 8. Plots of the oxygen permeation fluxes $J_{\mathrm{O}_{2}}$ vs $\left(P_{1} / P_{0}\right)^{n}-\left(P_{2} /\right.$ $\left.P_{0}\right)^{n}$ for the uncoated and LSC-coated hollow fiber membranes. $\left(P_{1}\right.$ was varied from 0 to $0.8 \mathrm{~atm}$.)

experimental data for the unmodified membrane at 1123 and $1173 \mathrm{~K}$ were 0.55 and 0.52 , which indicated that the unmodified membrane was predominantly controlled by the oxygen exchange reaction according to the theory of the ratecontrolling step, as described in eq 6. However, after surface modification, the $n$ values decreased to 0.35 and 0.30 at 1123 and $1173 \mathrm{~K}$, respectively, which means that after surface modification the oxygen permeation through the membrane was limited by both the surface exchange reaction and the bulk diffusion. This illustrates that a layer of catalytically porous, active LSC promotes the surface oxygen exchange reaction. This theoretical calculation coincided with the experimental results as described above.

To further interpret the above phenomenon, it is necessary to investigate the variation of the resistances of the unmodified and modified membranes. Zeng et al. ${ }^{35}$ reported that the total resistance of the oxygen permeation process can be calculated approximately from the oxygen permeation flux and the oxygen 
partial pressures on the feed and sweep sides according to the following expression:

$$
r_{\text {overall }}=\frac{R T}{4^{2} F^{2}} \frac{1}{A J_{\mathrm{O}_{2}}}\left(\ln \frac{P_{1}}{P_{2}}\right)
$$

where $r_{\text {overall }}$ is the total resistance for the oxygen transfer through the membrane. This formula is applicable to the following situations: (i) bulk diffusion is the main rate-limiting step; (ii) the surface oxygen exchange reaction on the feed side or the permeate side is the dominating rate-limiting step; and (iii) the surface exchange reaction and the bulk diffusion play similar important roles in the oxygen permeation process and cooperatively control the oxygen permeation process. The results calculated according to this theory of oxygen permeation resistance are displayed in Figure 9a. We can see that the

a)

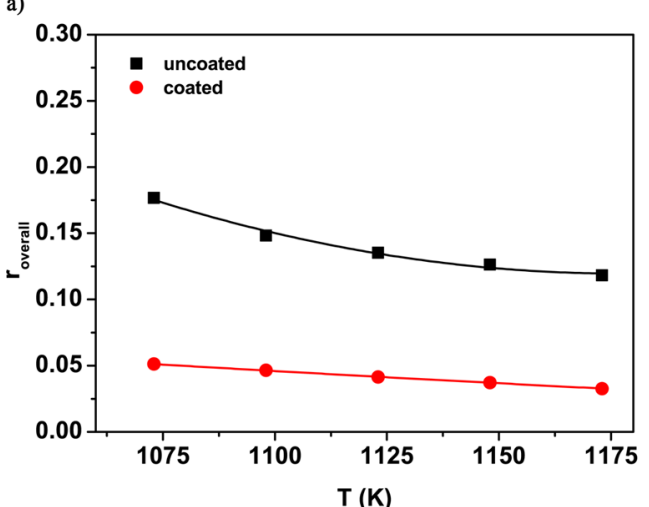

b)

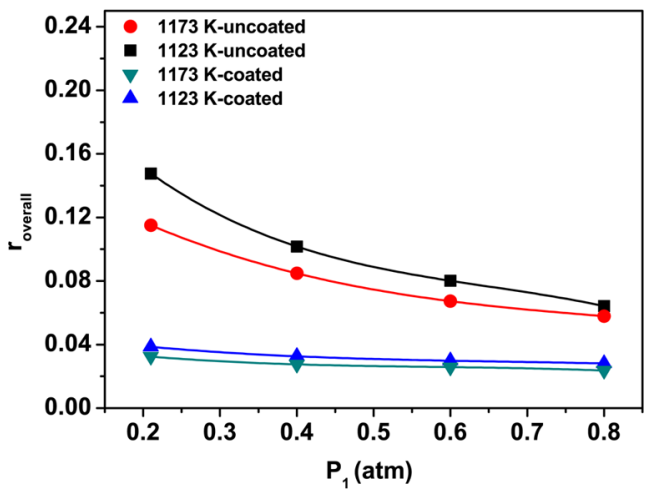

Figure 9. Oxygen permeation resistances of membranes pre- and postmodification (a) at different temperatures and (b) under different oxygen partial pressure and temperatures.

oxygen permeation resistance of the MCMHF membrane showed a noticeable decrease due to the surface modification. Figure $9 \mathrm{~b}$ illustrates the influence of the oxygen partial pressure on the value of $r_{\text {overall }}$ at different temperatures before and after the surface modification process. The decrease in $r_{\text {overall }}$ with increasing oxygen partial pressure on the feed side indicates that the oxygen transfer through the bare membrane is mainly controlled by the slow surface exchange reaction.

To show the effect of the coating thickness of the LSC porous layer on the oxygen permeation process, Figure 10 plots the oxygen permeation flux against the temperature for samples with different LSC layer thicknesses. We can see that when the thickness of the modified layer increased from 18 to $26 \mu \mathrm{m}$, the enhancement of the oxygen flux was only $0.14 \mathrm{~mL} \cdot \mathrm{cm}^{-2} \cdot \mathrm{min}^{-1}$.

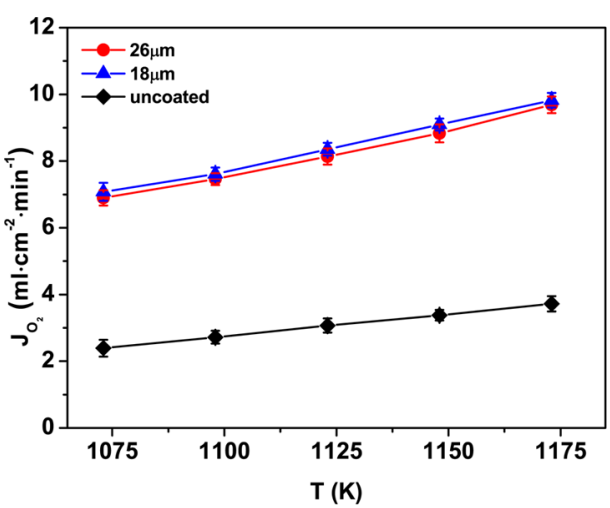

Figure 10. Effect of the LSC coating thickness on the oxygen permeation flux through the BSCF multichannel hollow fiber membranes.

The main reason for this phenomenon may be that as the thickness of the modified layer increased, some new resistance to the oxygen permeation process was introduced. For example, the thicker porous layer would produce more enclosed macrovoids, which would increase the length of the effective diffusion route ${ }^{36}$ and impede the bulk diffusion process. In addition, since only a slightly higher oxygen permeation flux was obtained, it is clearly uneconomical to increase the thickness of the modified layer to obtain such a small increase in the oxygen permeation flux.

An oxygen permeation membrane with promising industrial applications should possess both high oxygen permeation flux and good stability for long-term operation. Figure 11 shows the

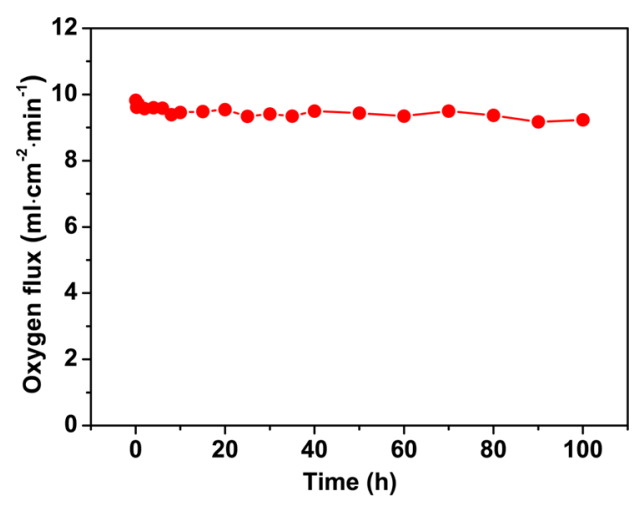

Figure 11. Time dependence of the oxygen permeation flux through an $18 \mu \mathrm{m}$ LSC-coated hollow fiber membrane. $\left(T=1173 \mathrm{~K}, F_{\text {air }}=120\right.$ $\left.\mathrm{mL} \cdot \mathrm{min}^{-1}, F_{\mathrm{He}}=80 \mathrm{~mL} \cdot \mathrm{min}^{-1}\right)$.

oxygen permeation flux of the LSC-modified MCMHF membrane as a function of time at $1173 \mathrm{~K}$ with an air flux of $120 \mathrm{~mL} \cdot \mathrm{min}^{-1}$ and a helium flux of $80 \mathrm{~mL} \cdot \mathrm{min}^{-1}$. A generally steady oxygen permeation flux of approximately $9.48 \mathrm{~mL} \cdot \mathrm{cm}^{-2}$. $\min ^{-1}$ was obtained at $1173 \mathrm{~K}$ during $100 \mathrm{~h}$ of operation. The result indicates that the LSC-modified MCMHF membrane possesses high oxygen permeation flux and good stability during the oxygen permeation test.

\section{CONCLUSIONS}

A porous-LSC-modified layer was successfully prepared on the surface of $\mathrm{Ba}_{0.5} \mathrm{Sr}_{0.5} \mathrm{Co}_{0.8} \mathrm{Fe}_{0.2} \mathrm{O}_{3-\delta}$ MCMHF membranes by combining spin-spraying and sintering techniques. The LSCmodified membranes exhibited noticeable enhancement in 
comparison with the membranes without surface modification. At $1173 \mathrm{~K}$, the oxygen permeation flux of the LSC-modified membrane reached as high as $9.68 \mathrm{~mL} \cdot \mathrm{cm}^{-2} \cdot \mathrm{min}^{-1}$. Moreover, when the oxygen partial pressure on the feed side was increased to $0.8 \mathrm{~atm}$, the oxygen permeation flux dramatically reached $20.1 \mathrm{~mL} \cdot \mathrm{cm}^{-2} \cdot \mathrm{min}^{-1}\left(F_{\mathrm{He}}=80 \mathrm{~mL} \cdot \mathrm{min}^{-1}\right)$, which is very promising in the production of pure oxygen. Meanwhile, experimental data and theoretical calculations suggested that the oxygen transfer through the membrane was limited by both the bulk diffusion process and the surface oxygen exchange reaction for the LSC-modified membranes. Our work demonstrates that surface modification is a very effective method for enhancing the oxygen permeation fluxes of MCMHF membranes.

\section{AUTHOR INFORMATION}

\section{Corresponding Author}

*Tel.: +86-25-83172266. Fax: +86-25-83172292. E-mail: wqjin@njtech.edu.cn.

\section{Notes}

The authors declare no competing financial interest.

\section{ACKNOWLEDGMENTS}

This work was supported by the Innovative Research Team Program by the Ministry of Education of China (IRT13070) and the Project of Priority Academic Program Development of Jiangsu Higher Education Institutions (PAPD).

\section{NOMENCLATURE}

\section{Variables}

$D_{\mathrm{v}}=$ vacancy diffusion coefficient of oxygen

$F=$ Faraday constant

$J_{\mathrm{O}_{2}}=$ oxygen permeation flux

$L=$ membrane thickness

$L_{\mathrm{C}}=$ characteristic membrane thickness

$P_{1}=$ oxygen partial pressure on the feed side

$P_{2}=$ oxygen partial pressure on the sweep side

$R=$ gas constant

$r_{\text {overall }}=$ total transport resistance

$V_{\mathrm{m}}=$ perovskite molar volume

$V_{\mathrm{O}}^{\infty}=$ concentration of oxygen vacancies

\section{Greek Symbols}

$\sigma_{\mathrm{e}}=$ electronic conductivity

$\sigma_{\mathrm{i}}=$ ionic conductivity

$\sigma_{\mathrm{i}}^{0}=$ ionic conductivity when the oxygen partial pressure is 1 atm

\section{REFERENCES}

(1) Sunarso, J.; Baumann, S.; Serra, J. M.; Meulenberg, W. A.; Liu, S.; Lin, Y. S.; da Costa, J. C. D. Mixed ionic-electronic conducting (MIEC) ceramic-based membranes for oxygen separation. J. Membr. Sci. 2008, 320 (1-2), 13-41.

(2) Pan, H.; Li, L.; Deng, X.; Meng, B.; Tan, X.; Li, K. Improvement of oxygen permeation in perovskite hollow fibre membranes by the enhanced surface exchange kinetics. J. Membr. Sci. 2013, 428, 198204.

(3) Wang, H.; Kölsch, P.; Schiestel, T.; Tablet, C.; Werth, S.; Caro, J. Production of high-purity oxygen by perovskite hollow fiber membranes swept with steam. J. Membr. Sci. 2006, 284 (1-2), 5-8.

(4) Joo, J. H.; Yun, K. S.; Lee, Y.; Jung, J.; Yoo, C.-Y.; Yu, J. H. Dramatically Enhanced Oxygen Fluxes in Fluorite-Rich Dual-Phase Membrane by Surface Modification. Chem. Mater. 2014, 26 (15), 4387-4394.
(5) Zhang, Z.; Chen, D.; Gao, Y.; Yang, G.; Dong, F.; Chen, C.; Ciucci, F.; Shao, Z. A CO 2 -tolerant nanostructured layer for oxygen transport membranes. RSC Adv. 2014, 4 (49), 25924-25932.

(6) Jiang, H.; Wang, H.; Werth, S.; Schiestel, T.; Caro, J. Simultaneous Production of Hydrogen and Synthesis Gas by Combining Water Splitting with Partial Oxidation of Methane in a Hollow-Fiber Membrane Reactor. Angew. Chem., Int. Ed. 2008, 47 (48), 9341-9344.

(7) Zhu, N.; Dong, X.; Liu, Z.; Zhang, G.; Jin, W.; Xu, N. Toward highly-effective and sustainable hydrogen production: Bio-ethanol oxidative steam reforming coupled with water splitting in a thin tubular membrane reactor. Chem. Commun. 2012, 48 (57), 7137-7139.

(8) Wei, Y.; Yang, W.; Caro, J.; Wang, H. Dense ceramic oxygen permeable membranes and catalytic membrane reactors. Chem. Eng. J. 2013, 220, 185-203.

(9) Shao, Z. P.; Haile, S. M. A high-performance cathode for the next generation of solid-oxide fuel cells. Nature 2004, 431 (7005), 170173.

(10) Zhou, W.; Ran, R.; Shao, Z. Progress in understanding and development of $\mathrm{Ba}_{0.5} \mathrm{Sr}_{0.5} \mathrm{Co}_{0.8} \mathrm{Fe}_{0.2} \mathrm{O}_{3-\delta}$-based cathodes for intermediate-temperature solid-oxide fuel cells: A review. J. Power Sources 2009, 192 (2), 231-246.

(11) Han, D.; Sunarso, J.; Tan, X.; Yan, Z.; Liu, L.; Liu, S. Optimizing Oxygen Transport through $\mathrm{La}_{0.6} \mathrm{Sr}_{0.4} \mathrm{Co}_{0.2} \mathrm{Fe}_{0.8} \mathrm{O}_{3-\delta}$ Hollow Fiber by Microstructure Modification and $\mathrm{Ag} / \mathrm{Pt}$ Catalyst Deposition. Energy Fuels 2012, 26 (8), 4728-4734.

(12) Yoon, J. S.; Yoon, M. Y.; Lee, E. J.; Moon, J.-W.; Hwang, H. J. Influence of $\mathrm{Ce}_{0.9} \mathrm{Gd}_{0.1} \mathrm{O}_{2-\delta}$ particles on microstructure and oxygen permeability of $\mathrm{Ba}_{0.5} \mathrm{Sr}_{0.5} \mathrm{Co}_{0.8} \mathrm{Fe}_{0.2} \mathrm{O}_{3-\delta}$ composite membrane. Solid State Ionics 2010, 181 (29-30), 1387-1393.

(13) Leo, A.; Liu, S.; da Costa, J. C. D. Production of pure oxygen from BSCF hollow fiber membranes using steam sweep. Sep. Purif. Technol. 2011, 78 (2), 220-227.

(14) Haworth, P.; Smart, S.; Glasscock, J.; da Costa, J. C. D. Yttrium doped BSCF membranes for oxygen separation. Sep. Purif. Technol. 2011, 81 (1), 88-93.

(15) Liu, S.; Tan, X.; Shao, Z.; da Costa, J. C. D. $\mathrm{Ba}_{0.5} \mathrm{Sr}_{0.5} \mathrm{Co}_{0.8} \mathrm{Fe}_{0.2} \mathrm{O}_{3-\delta}$ ceramic hollow-fiber membranes for oxygen permeation. AIChE J. 2006, 52 (10), 3452-3461.

(16) Kida, T.; Ninomiya, S.; Watanabe, K.; Yamazoe, N.; Shimanoe, K. High Oxygen Permeation in $\mathrm{Ba}_{0.95} \mathrm{La}_{0.05} \mathrm{FeO}_{3-\delta}$ Membranes with Surface Modification. ACS Appl. Mater. Interfaces 2010, 2 (10), 28492853.

(17) Hayamizu, Y.; Kato, M.; Takamura, H. Effects of surface modification on the oxygen permeation of $\mathrm{Ba}_{0.5} \mathrm{Sr}_{0.5} \mathrm{Co}_{0.8} \mathrm{Fe}_{0.2} \mathrm{O}_{3-\delta}$ membrane. J. Membr. Sci. 2014, 462, 147-152.

(18) Tan, X.; Wang, Z.; Liu, H.; Liu, S. Enhancement of oxygen permeation through $\mathrm{La}_{0.6} \mathrm{Sr}_{0.4} \mathrm{Co}_{02} \mathrm{Fe}_{0.8} \mathrm{O}_{3-\delta}$ hollow fibre membranes by surface modifications. J. Membr. Sci. 2008, 324 (1-2), 128-135.

(19) Leo, A.; Liu, S.; da Costa, J. C. D. The enhancement of oxygen flux on $\mathrm{Ba}_{0.5} \mathrm{Sr}_{0.5} \mathrm{Co}_{0.8} \mathrm{Fe}_{0.2} \mathrm{O}_{3-\delta}$ (BSCF) hollow fibers using silver surface modification. J. Membr. Sci. 2009, 340 (1-2), 148-153.

(20) Zhu, J.; Dong, Z.; Liu, Z.; Zhang, K.; Zhang, G.; Jin, W. Multichannel mixed-conducting hollow fiber membranes for oxygen separation. AIChE J. 2014, 60 (6), 1969-1976.

(21) Zhu, J.; Liu, Z.; Guo, S.; Jin, W. Influence of permeation modes on oxygen permeability of the multichannel mixed-conducting hollow fibre membrane. Chem. Eng. Sci. 2015, 122, 614-621.

(22) Adler, S. B. Mechanism and kinetics of oxygen reduction on porous $\mathrm{La}_{1-x} \mathrm{Sr}_{x} \mathrm{CoO}_{3-\delta}$ electrodes. Solid State Ionics 1998, 111 (1-2), $125-134$.

(23) van der Haar, L. M.; den Otter, M. W.; Morskate, M.; Bouwmeester, H. J. M.; Verweij, H. Chemical Diffusion and Oxygen Surface Transfer of $\mathrm{La}_{1-x} \mathrm{Sr}_{x} \mathrm{CoO}_{3-\delta}$ Studied with Electrical Conductivity Relaxation. J. Electrochem. Soc. 2002, 149 (3), J41-J46.

(24) Liu, Z.; Zhang, G.; Dong, X.; Jiang, W.; Jin, W.; Xu, N. Fabrication of asymmetric tubular mixed-conducting dense membranes by a combined spin-spraying and co-sintering process. J. Membr. Sci. 2012, 415, 313-319. 
(25) Zhang, C.; Chang, X.; Dong, X.; Jin, W.; Xu, N. The oxidative stream reforming of methane to syngas in a thin tubular mixedconducting membrane reactor. J. Membr. Sci. 2008, 320 (1-2), 401406.

(26) Leo, A.; Smart, S.; Liu, S.; da Costa, J. C. D. High performance perovskite hollow fibres for oxygen separation. J. Membr. Sci. 2011, 368 (1-2), 64-68.

(27) Wei, Y.; Liu, H.; Xue, J.; Li, Z.; Wang, H. Preparation and Oxygen Permeation of U-Shaped Perovskite Hollow-Fiber Membranes. AIChE J. 2011, 57 (4), 975-984.

(28) Wei, Y. Y.; Tang, J.; Zhou, L. Y.; Li, Z.; Wang, H. H. Oxygen Permeation through U-Shaped $\mathrm{K}_{2} \mathrm{NiF}_{4}$-Type Oxide Hollow-Fiber Membranes. Ind. Eng. Chem. Res. 2011, 50 (22), 12727-12734.

(29) Lin, Y. S.; Wang, W. J.; Han, J. H. Oxygen permeation through thin mixed-conducting solid oxide membranes. AIChE J. 1994, 40 (5), 786-798.

(30) Bouwmeester, H.; Kruidhof, H.; Burggraaf, A. Importance of the surface exchange kinetics as rate limiting step in oxygen permeation through mixed-conducting oxides. Solid State Ionics 1994, 72, 185194.

(31) Tenelshof, J. E.; Bouwmeester, H. J. M.; Verweij, H. Oxidative coupling of methane in a mixed-conducting perovskite membrane reactor. Appl. Catal., A 1995, 130 (2), 195-212.

(32) Hong, W. K.; Choi, G. M. Oxygen permeation of BSCF membrane with varying thickness and surface coating. J. Membr. Sci. 2010, 346 (2), 353-360.

(33) Huang, K. Q.; Goodenough, J. B. Oxygen permeation through cobalt-containing perovskites-Surface oxygen exchange vs. lattice oxygen diffusion. J. Electrochem. Soc. 2001, 148 (5), E203-E214.

(34) Liao, Q.; Zheng, Q.; Xue, J.; Wei, Y.; Wang, H. U-Shaped $\mathrm{BaCo}_{0.7} \mathrm{Fe}_{0.2} \mathrm{Ta}_{0.1} \mathrm{O}_{3-\delta}$ Hollow-Fiber Membranes with High Permeation for Oxygen Separation. Ind. Eng. Chem. Res. 2012, 51 (46), $15217-15223$

(35) Zeng, P. Y.; Chen, Z. H.; Zhou, W.; Gu, H. X.; Shao, Z. P.; Liu, S. M. Re-evaluation of $\mathrm{Ba}_{0.5} \mathrm{Sr}_{0.5} \mathrm{Co}_{0.8} \mathrm{Fe}_{0.2} \mathrm{O}_{3-\delta}$ perovskite as oxygen semi-permeable membrane. J. Membr. Sci. 2007, 291 (1-2), 148-156. (36) Zydorczak, B.; Li, K.; Tan, X. Mixed conducting membranesMacrostructure related oxygen permeation flux. AIChE J. 2010, 56 (12), 3084-3090 